\title{
Economics
}

\section{Evaluating the Effectiveness of the Investment Project}

\author{
Bakytkan Dauletbakov, ${ }^{1, ~, ~ G a l y m ~ D a u l e t b a k o v ² ~}$ \\ ${ }^{1}$ High School of Economics and Business Department, the Al-Farabi Kazakh National University, Almaty, Republic of Kazakhstan \\ ${ }^{2}$ LLP VILED, Almaty, Republic of Kazakhstan
}

Email address:

dauletbakovb@mail.ru (B. Dauletbakov)

\section{To cite this article:}

Bakytkan Dauletbakov, Galym Dauletbakov. Evaluating the Effectiveness of the Investment Project. Economics.

Vol. 4, No. 4, 2015, pp. 57-63. doi: 10.11648/j.eco.20150404.11

\begin{abstract}
Evaluating the effectiveness of investment projects is a prerequisite for the selection of the investment portfolio generated by the investor, and based on a number of traditional criteria: NPV (Net Present Value), IRR (Internal Rate of Return), PP (Payback Period), PI (Profitability index) and NFV (Net Future Value). We propose a model of multi-criteria decision-making on the selection of a portfolio of investment projects selected in terms of their effectiveness, based on the modification of the classical NPV (Net Present Value). In particular, the features and options compared NFV (Net Future Value) - efficiency test. In this direction, we should expect interesting results from a scientific and practical point of view. Our approach to assessing the effectiveness of investment projects based on the method of selection of optimal projects taking into account the set of criteria, intended to build interactive decision-making procedures, as the possibility of using rigorous methods, and knowledge and experience of the decision maker (DM). There are various approaches to selecting the resulting unnormalized preference relations on the basis of a plurality of predetermined. The simplest of these is the approach based on the principle of Borda that we have proposed in [17]. In this article we provide a description and the main approaches to the selection criteria by which assesses the effectiveness of possible variants of the portfolio of investment projects. It uses the methodology of constructing a set of solutions based on the Pareto principle.
\end{abstract}

Keywords: Criterion, Multicriteriality, Efficiency, Portfolio, Investment Projects, Decision Maker, The Pareto Solutions

\section{Introduction}

Evaluating the effectiveness of innovative projects is a prerequisite for the selection of the investment portfolio, which is formed by the investor, and is based on a number of criteria. To these, first of all, should include the payback period, internal rate of return, net present value and profitability index and net future value [1]-[5].

Today the market has a fairly wide range of computer programs for the calculation and comparative analysis of investment projects, both domestic and foreign developers $[6,7]$ (software for financial and economic evaluation of investment projects). Despite the variety of software products, there is the problem of formation and selection of the optimal set of investment projects, as all the above systems do not allow determining the optimal level of investment and does not take into account the dynamics of the firms.

A new method for selection of optimal projects taking into account the set of performance criteria is very desirable, and they are of great scientific and practical interest.

The impetus for the development of the methodology developed in this work was the desire to provide a computationally efficient, convenient and transparent way to calculate the most efficient investment projects in a given set of projects based on financial performance of the set of criteria understandable for the user. The theoretical novelty of the proposed methodology is that we offer a method for determining the Pareto frontier, which describes the need, or rather the only foreseeable number of alternatives for future use $[8,9]$.

The following key assumptions are used in our approach:

1) Introduction of a global or generalized quality criterion. There are two stages of its solution: the definition of the Pareto set and the search for solutions in this set.

2) We confine ourselves to man-machine method involving decision-makers.

3) Global function will be assessed by a scalar function or set the desired decision-makers in all local criteria. Then there is the following optimization problem: select from a variety of Pareto optimal solutions is a solution which most suits the decision maker.

The rest of the article is organized as follows. Section 2 
provides the justification and the choice of performance criteria. Section 3 describes a new method of selecting optimal projects taking into account the set of performance criteria. Section 4 provides an illustrative example of the application of effective procedures for the selection of investment projects and reports on the results of the experiment. Section 5 concludes.

In this paper we present the main approaches to the selection criteria by which assess the effectiveness of admissible portfolio of investment projects. Proposed formulation of the problem of choosing the rational version of the feasible set of portfolio, which is reduced to the problem of decision making in many quality criteria. The main attention is paid to the development of a methodology for choosing a rational variant of the set of feasible projects based on many criteria of optimality. Application of the method is illustrated with real examples.

\section{Justification and Selection Criteria of Efficiency}

Issues on improving the efficiency project portfolios at this stage are highly relevant both in theoretical and practical terms [10-12].

From a theoretical point of view the problem is that the portfolio estimated on the basis of classical indicators such as for example NPV, IRR, PP and many others. However, these indicators have many drawbacks [12]. None of the above criteria by itself is not sufficient for the adoption of the project. Each of the methods of analysis of investment projects makes it possible to consider only some of the characteristics of the billing period, to find out the important points and details. Therefore for a comprehensive evaluation is necessary to use all of these criteria, including net future value (NFV) in the aggregate.

In this work [13], proposed new methods of evaluation of social projects, the model of which is represented as a set of interrelated business processes (business process portfolio). On the basis of the process models are laid streaming model of works, resources, finance, and etc., lying on the basis of representation of social projects. Many projects (planned or implemented) form a portfolio of projects. Efficiency of the project portfolio is proposed to estimate using the compounding operation of financial flows projects.

In this paper, issues of optimization efficiency of the project portfolio is decided based on the results simulation modeling obtained on the basis of the implementation of a set of experiments, interconnected business processes. Each of the simulation experiments is a variant of the structural organization of clusters of projects - network model. It is assumed that the various embodiments of business processes in this system are defined by the user.

Thus, there is a predetermined set of variants of business processes at the network level. Each set of options for business processes under investigation on the network model. A result of modeling is calculated flows of projects cluster of each option. Required at the base of some entered preference relations order a given set of options for business processes and identify the best. I.e. there is a problem of choice or decision of a given set of admissible.

Formally, the problem of optimizing the efficiency of the project portfolio in general is reduced to an extreme problem:

$$
F(x) \rightarrow \underset{x^{i} \in \Omega}{o p t},
$$

Where with the position of the system approach is necessary to establish

$F(x)$ - Content evaluation criteria options for portfolio efficiency projects;

Argument $x$ - kind of variable parameters;

$\Omega$ - Range of permissible values of variable parameters;

Operator opt - selected principle of optimality.

Note that from the above is the case when $\Omega=\left\{x^{i}\right\}$ - we have a given set of options for the effectiveness of the project portfolio, each of which is subjected to investigation of model simulations.

$x^{i} \in \Omega$ - Some $i$ variant of the system;

$$
|\Omega|=m, i=\overline{1, m},
$$

Where $m$ - quantity of the variants.

Each of the options is estimated by the vector quality criterion:

$$
f\left(x^{i}\right)=\left(f_{1}\left(x^{i}\right), f_{2}\left(x^{i}\right), \ldots, f_{n}\left(x^{i}\right)\right)
$$

Where $f_{k}(x)-\kappa$ is the local criteria of quality, $k=\overline{1, n}$.

As noted, the local quality criterion identified some characteristics of the system. For example, $f_{1}$ - net present value, $f_{2}$ - Internal Rate of Return, $f_{3}$ - profitability index, $f_{4}$ - payback period, $f_{5}$ - net future value will be called the vector criterion by which alternatives are evaluated, i.e. investment projects.

Further, without loss of generality, we assume that you want to achieve, perhaps smaller values of all the components of the vector criterion, i.e. minimize the vector function (3).

Thus, there is a problem of vector or multi-criteria optimization. As we know the general solution of this problem is the set of Pareto optimal solutions - $M_{P}$. This set is called in the literature as well: lots of subordinates, nondominated or efficient solutions, negotiation or compromise set. Recall that the decision $x^{i}$ called Pareto optimal if the admissible region there is no solutions $x^{k} \in \Omega$, to satisfy:

$$
f_{j}\left(x^{k}\right) \leq f_{j}\left(x^{i}\right), j=\overline{1, n}
$$

Where at least one of the inequalities must be strict.

All decisions Pareto form a set $M_{P}$.

Note these features of the problem (3). Evaluation of efficiency of each option the project portfolio is made on set 
of parameters. This leads to the release set $M_{P} \subset \Omega$, which in general is much "smaller" area of feasible solution $\Omega$. Elements of this set have objective property cannot simultaneously improve all components of the vector criterion. I.e. transition from one to another effective option is accompanied by improvement of the components of the vector criterion at the expense of the rest.

\section{Description of a New Method for Selecting the Optimum Projects Taking Into Account the Set of performance Criteria}

In paragraph 2, the task of choosing the most effective innovative project in a given set (package) projects based on many criteria of financial efficiency. The general solution of multi-criterion optimization, as is well known, is the set of solutions, Pareto optimal. For the case given set of course, but if the initial feasible set of criteria is sufficient volume, the number of financial and effective solutions can also be large. While you need to choose a unique or sufficiently foreseeable number of alternatives, which are recommended for use.

Common approach to solving this problem is the introduction of a global or generalized criterion of quality. The mathematical formulation of the problem in this case is as follows:

$$
F(x) \rightarrow \min _{x \in M_{p}}
$$

Where $M_{p}$ - multiple Pareto, selected from the allowable region $\Omega$ on the basis of vector criteria $f(x)$ from the formula (3).

Generalized criterion can be given different meanings depending upon the specifics of the problem, and there are numerous ways to build on the scalarization of the vector criterion $[14,15]$. Sometimes, especially in the construction dialog procedures of decision making, this criterion is interpreted as a utility function of the person responsible for the decision maker (DM), which can make judgments about the degree of preference of a particular variant of the investment project. We further assume that the function is defined, or for any option calculate its value, for example, by imitation.

Address the problem (5) can be divided into two stages of its solution: the definition of the Pareto and search solutions in this set. The first step amenable to formalization, while for the second phase is necessary to introduce some additional axiomatic system or preferences. Often in the second stage is implemented search for the best solutions in the Pareto set from the viewpoint of the person responsible for making decisions (DMP). It specifies the set of criteria, the values of which need to be improved, should not deteriorate and are not significant.

Basis of the model by which decisions are made, is the matrix of solutions D:

$$
D=\left[\begin{array}{l}
f_{1}\left(x^{1}\right), f_{2}\left(x^{1}\right), \ldots, f_{n}\left(x^{1}\right) \\
f_{1}\left(x^{2}\right), f_{2}\left(x^{2}\right), \ldots, f_{n}\left(x^{2}\right) \\
\ldots \ldots \ldots \ldots \ldots \ldots \ldots \ldots \ldots \ldots \ldots \ldots \\
f_{1}\left(x^{m}\right), f_{2}\left(x^{m}\right), \ldots, f_{n}\left(x^{m}\right)
\end{array}\right] .
$$

In this matrix, each row is associated with the certain embodiments, a column - the corresponding figure. At the intersection of $\mathrm{i}$ - row and the $\mathrm{j}$ - column is the value of $\mathrm{j}-$ criterion for $\mathrm{i}$ - variant, and this value can be both quantitative and qualitative. After building the solution matrix finding Pareto (efficient) solutions by specially organized search of all possible solutions and their pair wise comparisons [16]. This procedure is quite effective even when a large number of options and criteria. If the resulting set $M_{p}$ is a small number of elements, the DMP by simple comparison based on experience and expertise is able to identify the most preferred. In cases where the effective set $M_{p}$ is little different from a number of preset options, it is necessary and it is necessary to resort to formal methods obtain a unique solution.

There are many approaches to solving multi-objective problems, which are used in its basis the idea of scalarization vector criterion. Sufficient review of these methods is given in [13-16]. However, the most promising and evolving approach to solving these problems is to build interactive decision-making procedures, the possibility of using both rigorous methods and knowledge, experience DMP [8-9, $15,16]$.

Here we consider the possibility of solving the problem (5) man-machine method involving decision-makers. [9] Global function $\mathrm{F}(\mathrm{x})$ will be assessed by a scalar function of the form:

$$
\Phi(x)=\rho(f(x), \tilde{f})
$$

Where $\rho$ - distance to some chosen metric;

$f(x)$ - Vector function, which must be minimized;

$\tilde{f}$ - n- dimensional vector, which is taken as a goal or "ideal" point for the vector function $f$.

Note that in the expression (7) vector gives meaning vector levels desired or set DMP all local criteria. Then we have the following optimization problem: select from a variety of Pareto optimal solutions of a solution that suits the largestDMP according to criterion (7).

The procedure for selecting solutions for vector quality criteria for the task (5) includes the following stages:

Stage 1. DMP is given (fixed) vector desired levels:

$$
\tilde{f}=\left(\tilde{f}_{1}, \tilde{f}_{2}, \ldots, \tilde{f}_{n}\right) .
$$

$\tilde{f}$ In cases where the number of local criteria large DMP may experience serious difficulties to define the vector. Then we can use a vector: 


$$
f^{0}=\left(f_{1}^{0}, f_{2}^{0}, \ldots, f_{n}^{0}\right),
$$

Each component of which corresponds to the minimum of the corresponding local criterion in the admissible region, i.e.

$$
f^{0} f_{j}^{0}=\min _{x^{i} \in \Omega} f_{j}\left(x^{i}\right), \quad j=\overline{1, n} .
$$

Vector in the general case does not belong to the set of values of the vector criterion for acceptable solutions $x^{i} \in \Omega$, but can serve as «ideal» ratings for the appointment of the DM desired levels for each of the local criteria. The fact that the largest vector component $f^{0}$ DMP can judge about the maximum possible effect on each of the criteria.

Stage 2. Solve the problem of determining the maximum of the expression (7) on the set of solutions, Pareto optimal, i.e.

$$
\Phi(x)=\rho(f(x), \tilde{f}) \rightarrow \min _{x^{i} \in M_{p}}
$$

Thus determined decision $x^{i_{s}}$ is that

$$
\Phi\left(x^{i_{k}}\right)=\min _{x^{i} \in M_{p}} \Phi(x)
$$

Stage 3. In point found $x^{i_{i}}$ calculates the value of the vector criterion

$$
f\left(x^{i_{*}}\right)=\left(f_{1}\left(x^{i_{*}}\right), f_{2}\left(x^{i_{\psi}}\right), \ldots, f_{n}\left(x^{i_{s}}\right)\right) .
$$

The result is presented DMP. Comparison of vectors $f\left(x^{i_{s}}\right)$ and $\tilde{f}$ may lead to further continue the process. If this result is not satisfied with DMP for some components or requirements for the functioning of the system studied were inflated, it can adjust the desired levels of vector $\tilde{f}$ and revert back to step 2. Perhaps also a change of the criterion $\Phi(x)$ expression in (7)

In terms of criteria $\Phi(x)$ can be used traditional metrics like:

$$
\begin{aligned}
& \Phi_{1}(x)=\sum_{j=1}^{n}\left[f_{j}(x)-\tilde{f}_{j}\right]^{2}, \\
& \Phi_{2}(x)=\sum_{j=1}^{n}\left|f_{j}(x)-\tilde{f}_{j}\right|, \\
& \Phi_{3}(x)=\max _{j=1, n}\left|f_{j}(x)-\tilde{f}_{j}\right| .
\end{aligned}
$$

If you know the comparative importance of local criteria or it can be expressed as a weight vector

$$
w\left(w_{1}, w_{2}, \ldots, w_{n}\right),
$$

Where

$$
w_{j} \geq 0, \quad \sum_{j=1}^{n} w_{j}=1
$$

then the expressions (10) - (12) as the corresponding components will multiplied by a weighting factor. Sometimes take a function scalarized criterion as a weighted sum of local criteria:

$$
\Phi(x)=\sum_{j=1}^{n} w_{j} f_{j}(x)
$$

where $w_{j}$ - defined (set) in accordance with $(13,14)$.

\section{Examples of Application of Effective Procedures for the Selection of Investment Projects}

To illustrate the application of the proposed method of selecting the most effective investment project on a given finite set of options is a simple example (example SKHPK "Plemzavod" Almaty "). The literature is quite wide application received two-criterion model "cost effectiveness." These models are known to be well illustrated by the corresponding graphs in the continuous case. For discrete mapping from the set of feasible solutions to the values of the criteria is a point character.

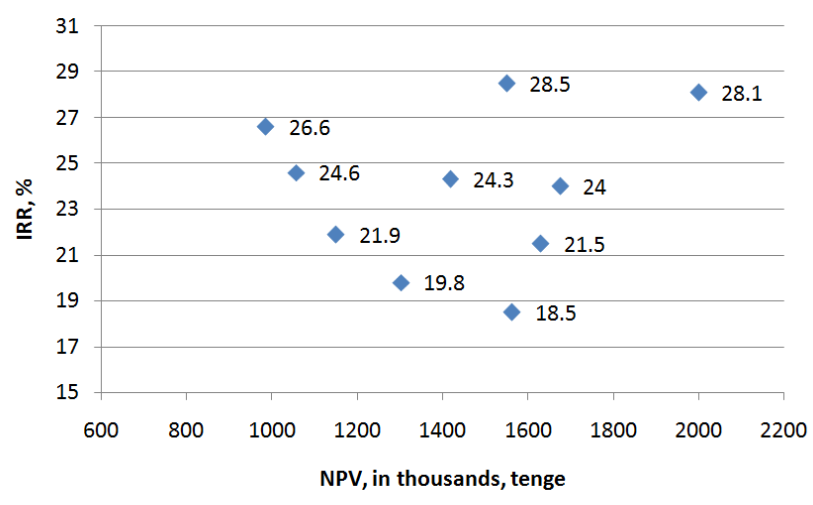

Fig. 1. The set of options in the space of criteria.

In Fig. 1 shows a conventional example (example SKHPK "Plemzavod" Almaty ") of displaying a plurality of options (number - 10) in the space of criteria $f_{1},-\mathrm{NPV}$, тыс.тенге, $f_{2}-$ IRR, \% . Supposed to minimize the component of the vector criterion. In Table 1 the project 2 corresponds to the value of the vector criterion $f\left(x^{2}\right)=\left(f_{1}\left(x^{2}\right), f_{2}\left(x^{2}\right)\right)$. From this example, efficient (Pareto) solutions are the options 4, 10, $2,9,7$, i.e.

$$
M_{P}=\left\{x^{4}, x^{10}, x^{2}, x^{9}, x^{7}\right\}
$$

And now many of you want to select only some. The criteria $f_{1}, f_{2}$ can be interpreted, respectively, as «NPV(i) IRR».

Consider the following example. Suppose we are given a variety of options $\Omega$, consisting of 10 variants, i.e. 


$$
\Omega=\left\{x^{1}, x^{2}, \ldots, x^{10}\right\}
$$

each of which is estimated vector criterion of 5 - component:
Table 1 shows the relevant matrix of solutions to this embodiment, in which $\mathrm{i}$ - row corresponds to the values of the vector criterion for option i, i.e. $f\left(x^{i}\right)$.

$$
f(x)=\left(f_{1}(x), f_{2}(x), f_{3}(x), f_{4}(x), f_{5}(x)\right)
$$

\begin{tabular}{|c|c|c|c|c|c|}
\hline Investment projects & $f_{1}$ - NPV, thousand, tenge & $f_{2}-\mathbf{I R R}, \mathbf{\%}$ & $f_{3}$ - PP, years & $f_{4}-\mathbf{P I}$ & $f_{5}$ - NFV, thousand, tenge \\
\hline Project 1 & 1417 & 24,3 & 2,5 & 1,1 & 12373 \\
\hline Project 2 & 1150 & 21,9 & 1,3 & 1 & 10042 \\
\hline Project 3 & 1550 & 28,5 & 4,5 & 1,9 & 13535 \\
\hline Project 4 & 985,6 & 26,6 & 3,5 & 1 & 8606,3 \\
\hline Project 5 & 1675 & 24 & 3,2 & 1 & 14626 \\
\hline Project 6 & 2000 & 28,1 & 3,8 & 1,3 & 17464 \\
\hline Project 7 & 1562,3 & 18,5 & 3,5 & 1,2 & 13642 \\
\hline Project 8 & 1629,8 & 21,5 & 2,8 & 1 & 14231 \\
\hline Project 9 & 1301,5 & 19,8 & 2,9 & 1,1 & 11365 \\
\hline Project 10 & 1056,6 & 24,6 & 1,9 & 1,5 & 9226,3 \\
\hline
\end{tabular}

Table 1. Matrix solutions for selected investment projects.

The next stage is to set separation $M_{P}$ - effective options, which are based on sequential sorting options and pair wise comparison of the values of the vector criterion. For example, the project 1 preferred than Project 3, i.e. $x^{1} \succ x^{3}$ by the fact

$$
x^{2} \succ x^{3}, x^{4} \succ x^{6}, x^{5} \succ x^{6}, x^{7} \succ x^{6}, x^{8} \succ x^{6}, x^{9} \succ x^{6}, x^{10} \succ x^{6}, x^{2} \succ x^{1}, x^{8} \succ x^{5}
$$

Thus revealed many effective solutions, which consists of the following options:

The corresponding matrix of a plurality of solutions is presented in Table 2 . that $f_{j}\left(x^{1}\right) \leq f_{j}\left(x^{3}\right), j=\overline{1,5}$. Similarly, too much is made,

\begin{tabular}{|c|c|c|c|c|c|}
\hline Investment projects & $f_{1}$ - NPV, thousand, tenge & $f_{2}-\mathbf{I R R}, \%$ & $f_{3}$ - PP, years & $f_{4}-\mathbf{P I}$ & $f_{5}$ - NFV, thousand, tenge \\
\hline Project 2 & 1150 & 21,9 & 1,3 & 1 & 10042 \\
\hline Project 4 & 985,6 & 26,6 & 3,5 & 1 & 8606,3 \\
\hline Project 7 & 1562,3 & 18,5 & 3,5 & 1,2 & 13642 \\
\hline Project 8 & 1629,8 & 21,5 & 2,8 & 1 & 14231 \\
\hline Project 9 & 1301,5 & 19,8 & 2,9 & 1,1 & 11365 \\
\hline Project 10 & 1056,6 & 24,6 & 1,9 & 1,5 & 9226,3 \\
\hline
\end{tabular}
we can write:

$$
M_{P}=\left\{x^{2}, x^{4}, x^{7}, x^{8}, x^{9}, x^{10}\right\}
$$

Table 2. Matrix of many effective solutions investment projects.

Options outlined in the Table. 2 are not comparable among themselves by vector criterion. Every pair wise comparison of options shows that one of the indicators of the preferred option, and through the remainder of indicators - the other. Therefore, to apply the single selection procedure embodiment set forth above.

Stage 1. Given the vector of desired levels

$$
\widetilde{f}=\left(\widetilde{f}_{1}, \widetilde{f}_{2}, \ldots, \widetilde{f}_{5}\right) .
$$

Suppose he is identified with the vector $f^{0}$, each component of which corresponds to the minimum of the corresponding local criterion, i.e. $\widetilde{f} \equiv f^{0}$.

In our example from table 2 
Thus, the solution of the problem (16) is $x^{4}$.

Stage 3. In these points $x^{4}$ calculated value of the vector criterion

$$
f\left(x^{4}\right)=(985,6 ; 26,6 ; 3,5 ; 1 ; 8606,3)
$$

The result is presented for the analysis of DMP. Assume that the result does not suit DMP, and it must take into account the importance of comparative criteria, which is reflected, for example, the weight vector of the form:

$$
w=\{0,6 ; 0,2 ; 0,1 ; 0,005 ; 0,005\} .
$$

Using the criteria of the form (16), but the weighted vector $w$, i.e.

$$
\Phi(x)=\sum_{i=1}^{5} w_{i}\left|f_{i}(x)-f_{i}^{0}\right|
$$

Again go to step 2.

Calculated values of the criterion at the points of $M_{P}$ :

$\Phi\left(x^{2}\right)=1195,66 ; \Phi\left(x^{4}\right)=1026,395 ; \Phi\left(x^{7}\right)=1622,39$;

$\Phi\left(x^{8}\right)=1693,06 ; \Phi\left(x^{9}\right)=1352,355 ; \Phi\left(x^{10}\right)=1098,96$

In this case, the solution is an option $x^{4}$.

Indeed, for this embodiment is characterized by the lowest values of the criteria $f_{1}, f_{2}$, which corresponds to a given comparative importance of criteria. The newly calculated value of the vector criterion for option $x^{4}$ :

$$
f\left(x^{4}\right)=(985,6 ; 26,6 ; 3,5 ; 1 ; 8606,3) .
$$

Thus, depending on the specific system investigated by changing the importance of comparative criteria, metric and select the "ideal" criteria for points in space, it is possible by providing an interactive procedure to allocate a plurality of effective solutions $M_{P}$ identify the most preferred option for DMP. However, the resulting set of efficient solutions of investment projects is not unique. This example was obtained optimal set of investment projects from the point of view of one expert (DMP).

To obtain more objective result advisable to involve more experts, with the result of the work of each will be constructed by comparing it with the effectiveness of the evaluation function values counted for each project.

On the basis of the calculation results obtained in the course of each expert, developed integral indicator for the assessed projects made their ranking and selects the optimal set of investment projects.

\section{Conclusion}

We have proposed a new approach to evaluating the effectiveness of investment projects based on the method of selection of optimal projects based on numerous criteria, which offers build interactive decision-making procedures, as the possibility of using rigorous methods, and knowledge and experience of the decision maker (DM).
In this article we provide a description and the main approaches to the selection criteria by which assesses the effectiveness of possible variants of the portfolio of investment projects. Here we the methodology of constructing a set of solutions based on the Pareto principle. It is easy to see that the Pareto principle does not ensure the uniqueness of the resulting preference relation and defines, in general, some of their family. This means that, adhering to Pareto principle, we get a family of trade-offs, in which will need to choose the best.

In the future researches it is expected to include consideration of additional criteria that evaluate investment projects in terms of their social, economic importance, etc. The need for inclusion of such criteria caused by consideration of the role of the state as the person, making decisions on the selection of investment projects aimed at developing the economic, social welfare.

\section{References}

[1] J. Van Horn. C. Basics of financial management / translated from English. M .: Finance and Statistics, 1997.

[2] Holt R.N. Fundamentals of Financial Management / translated from English. M .: Delo 1995.

[3] Financial management: Theory and Practice /E.S. Stoyanova. M .: Perspective, 1993.

[4] Sharpe W.F., Gordon J.A., Bailey I.V. Investments. N.Y .: PrenticeHall, 1995.

[5] Naumov A.A, Khodusov NV Management of portfolio investments. Models and algorithms. - Novosibirsk: Offset, 2005. - 298 p.

[6] http://www.unido.ru/resources/software/comfar.

[7] http://lib.sportedu.ru http://cfin.ru/management.

[8] Dauletbakov B.D. Applying the Pareto principle in the planning of agroindustrial formations. Proceedings of the conference of experts of the Commonwealth of Independent States - Alma-Ata, 1992. - p. 27 - 29.

[9] Dauletbakov B.D. Management decision-making in agricultural production (the theory, methodology, model): Monograph. Almaty, 2005. - 425 p.

[10] Dauletbakov D.B., Naumov A.A., Dauletbakov G.B. Methods of estimating financial performance of private business processes // Herald of the Kazakh National University. Economic Series, 2010, № 5 (81), pp 46-50.

[11] Dauletbakov D.B., Naumov A.A., Dauletbakov G.B. Assessing the effectiveness of financial and economic systems with the use of models in the form of business processes // Herald of the National Academy of Sciences of the Republic of Kazakhstan, 2010, № 4, pp 39-43.

[12] Dauletbakov D.B., Naumov A.A., Dauletbakov G.B. System formation and find the best portfolio of business processes // Herald of the National Academy of Sciences of the Republic of Kazakhstan, 2010, № 4, pp 44-48. 
[13] Naumov A.A., Dauletbakov B.D. Effectiveness of social projects. Proceedings of the International scientific and practical conference "Sustainable development of Kazakhstan's economy: modernization imperatives and business inzhiring." Almaty, Kazakhstan on 29-30 March 2012. KazNTU after KI Satpayev. Pp 383-388.

[14] Podinovskii Vladislav. Pareto-optimal solutions of multiobjective problems / VV Podinovskii, VD Nogin. - Ed. 2nd, rev. and add. - M.: FIZMATLIT, 2007. - 255 p.
[15] Larichev, O.I. Theory and methods of decision-making, and Chronicle in Magic countries : the textbook / O.I. Larichev. 2nd ed., Rev. and add. - Moscow: Logos, 2003. - 391 p.

[16] Ivanilov Y.P., Lotov A.V. Mathematical models of the economy. - M .: Science, 2007.

[17] Alexey An, Bakytkan Dauletbakov, Eugene Levner. Computational Linguistics and Intelligent Text Processing Lecture Notes in Computer Science Volume 8404, 2014, pp 404-416. 